\section{$\underset{\substack{\text { hommes } \\ \text { \& migrations }}}{ }$}

\section{Hommes \& migrations}

Revue française de référence sur les dynamiques

migratoires

$1285 \mid 2010$

L'appel du pied

\title{
Les effets symboliques des migrations dans le football de la Caraïbe
}

\author{
Harry P. Mephon
}

\section{(apenEdition \\ Journals}

\section{Édition électronique}

URL : http://journals.openedition.org/hommesmigrations/1179

DOI : 10.4000/hommesmigrations.1179

ISSN : 2262-3353

\section{Éditeur}

Musée national de l'histoire de l'immigration

\section{Édition imprimée}

Date de publication : 1 mai 2010

Pagination : 66-79

ISSN : 1142-852X

\section{Référence électronique}

Harry P. Mephon, « Les effets symboliques des migrations dans le football de la Caraïbe », Hommes \& migrations [En ligne], 1285 | 2010, mis en ligne le 29 mai 2013, consulté le 19 avril 2019. URL : http:// journals.openedition.org/hommesmigrations/1179; DOI : 10.4000/hommesmigrations.1179 


\section{Les effets symboliques des migrations dans le football de la Caraïbe}

Par Harry P. Mephon, sociologue

Marius Trésor, Thierry Henry, Lilian Thuram, William Gallas ou Nicolas Anelka : autant de noms rattachés aux plus belles pages de l'équipe de France de football ou à celles de grands clubs européens.

Le rôle et l'implication de ces talents dans l'équipe nationale résultent d'un long cheminement sociohistorique et de raisons complexes qui se situent hors des terrains, voire hors du territoire national. Peu d'analyses interrogent les inconscients collectifs et les conditions sociales en jeu. Car, entre la Carailbe et la France, le football est au cœur des migrations des populations et de leurs effets symboliques. 
Malgré la présence de trois Guadeloupéens dans l'équipe championne du monde en 1998, il a fallu qu'un avion s'écrase en 2005 au Venezuela pour qu'une équipe de France A, se déplace pour la première fois dans la Caraïbe, en Martinique, pour rendre un hommage mortuaire. Au-delà des émotions, les relations développées et entretenues entre les départements français de la Caraïbe et l'Hexagone ne permettent pas de penser la réalité des hommes impliqués dans le football guadeloupéen et martiniquais. En situant notre point de vue depuis la Caraibe, il s'agit de présenter les migrations des populations comme une condition déterminante dans la diffusion du football dans la Caraïbe francophone, en Haït nation déjà présente en Coupe du monde en 1934 -, en comparaison avec ce qui débute en Guadeloupe à la même époque.

La présence d'une communauté antillaise en France, qui trouve dans le sport un moyen d'affirmation sociale, sera appréhendée à partir de la transformation de sa dynamique migratoire et ses récentes significations dans les investissements de ses membres au sein de la sélection régionale de football, les Gwada Boys.

\section{La diffusion des sports à l'époque coloniale}

Les îles de la Caraïbe se situent au carrefour de flux migratoires importants, incessants et multidirectionnels, créant des phénomènes sociaux culturels d'une grande singularité. Les premières migrations son très anciennes. Elles débutent dès 5000 av. J.-C., avec les Arawak, une peuplade originaire de la région du bassin de l'Orénoque, dans l'actuel Venezuela. Ce sont les Taïnos, souvent appelés Caraïbes, un groupe arawak, qui entrent en contact avec les premiers conquérants européens. Ce contact est fatal aux indigènes - qui refusent la soumission et le travail forcé - et à leur culture. Ils sont exterminés progressivement.

$\mathrm{Au} \mathrm{XVI}^{\mathrm{e}}$ siècle, les îles à sucre captent des mouvements de population liés à la colonisation, qui repose sur le modèle économique des plantations de canne à sucre, nécessitant une importante main-d'ceuvre à bas prix. Nous sommes aux prémices des dynamiques de la mondialisation du système capitaliste, au moment où se joue la mondialisation des jeux traditionnels britanniques, qui à partir du XVII ${ }^{\mathrm{e}}$ siècle, prennent le nom de "sport", à l'exemple du cricket, du rugby, du base-ball et du football. La diffusion des jeux, loin de rester cantonnés à leur espace d'origine, s'adapte et s'insère dans les idéologies impériales, en particulier celle de la puissance britannique. Les premiers matchs de cricket se déroulent en 1806 dans la garnison de la Barbade; en 1865 le premier match intercolonial regroupe de riches 


\section{Les joueurs guadeloupéens, martiniquais et guyanais dans la sélection nationale de football}

Vingt-sept joueurs guadeloupéens, martiniquais, guyanais ou originaires de la Caraïbe ont été sélectionnés en équipe de France $A$.

Trois ont été champions du monde en 1998 : Henry, Diomède, Thuram

Quatre champions d'Europe en 2000 : Anelka, Henry, Thuram, Wiltord.

Douze joueurs titrés en Coupe des confédérations de 2001 à 2003.

Dix-neuf représentations en Coupe du monde de 1938 à 2006.

Douze représentations lors de l'Euro de 1992 à 2004.

\begin{tabular}{|c|c|c|c|c|}
\hline NOMS & Sélections & Buts & Années & Origine \\
\hline Raoul Diagne & 18 & 0 & $1931-1940$ & Guyane \\
\hline Xercès Louis & 12 & 0 & 1954-1956 & Martinique \\
\hline Paul Chillan & 2 & 0 & 1963 & Guadeloupe \\
\hline Daniel Charles - & 4 & 0 & 1964 & Guadeloupe \\
\hline Marius Trésor & 65 & 4 & $1971-1983$ & Guadeloupe \\
\hline Gérard Janvion & 40 & 0 & 1975-1982 & Martinique \\
\hline Alain Couriol & 12 & 2 & $1980-1983$ & Guadeloupe \\
\hline Luc Sonor & 9 & 0 & 1987-1989 & Guadeloupe \\
\hline Frank Sylvestre & 11 & 0 & 1989-1992 & Guadeloupe \\
\hline Jocelyn Angloma & 37 & 1 & $1990-1996$ & Guadeloupe \\
\hline Bernard Lama & 44 & 0 & $1993-2006$ & Guyane \\
\hline Jean-Pierre Cyprien & 1 & 0 & 1994 & Guadeloupe \\
\hline Lilian Thuram & 142 & 2 & 1994-2006 & Guadeloupe \\
\hline Thierry Henry & 142 & 51 & $1997-2010$ & Guadeloupe \\
\hline Nicolas Anelka & 14 & 6 & $1998-2010$ & Martinique \\
\hline Bernard Diomède & 8 & 0 & 1998 & Guadeloupe \\
\hline Sylvain Wiltord & 92 & 26 & $1999-2010$ & Guadeloupe \\
\hline Philippe Christanval & 6 & 0 & 2000-2002 & Guadeloupe \\
\hline Steve Marlet & 23 & 6 & 2000-2004 & Guadeloupe \\
\hline Mikaël Silvestre & 40 & 0 & 2001-2006 & Guadeloupe \\
\hline Olivier Dacourt & 21 & 1 & 2001-2004 & Guadeloupe \\
\hline William Gallas & 78 & 4 & 2002-2010 & Guadeloupe \\
\hline Éric Abidal & 45 & 0 & 2004-2005 & Guyane \\
\hline Louis Saha & 8 & 2 & 2004-2005 & Guadeloupe \\
\hline Jonathan Zebina & 1 & 0 & 2005 & Martinique \\
\hline Pascal Chimbonda & & 0 & 2006 & Guadeloupe \\
\hline Jimmy Briand & 3 & 0 & 2008 & Guadeloupe \\
\hline
\end{tabular}


planteurs et oppose une équipe de la Barbade et une du Guyana. Le fait colonial reste essentiel dans la compréhension de l'universalité des sports et de la diversité des pratiquants.

En ce qui concerne les colonies françaises, la Guadeloupe, la Martinique, trois grandes phases migratoires se succèdent: une phase de peuplement, une phase d'enracinement des populations sur les îles et, la dernière, une phase de "transbord", étape de rupture migratoire située en Europe, essentiellement en France.

La première, la phase de peuplement initié au départ de l'Europe, s'amorce au $\mathrm{XVII}^{\mathrm{e}}$ siècle et dure trois siècles. Cette période esclavagiste marquée par la traite correspond, après la disparition des Amérindiens, à des vagues continues d'immigration. Se succèdent des arrivées permanentes de colons libres blancs (les habitants), de travailleurs semi-contraints (les engagés) et d'esclaves. Ces derniers constituent une main-d'ceuvre forcée majoritaire en nombre, composée de populations noires d'Afrique et un peu plus tard, après la deuxième libération des esclaves (1848), de travailleurs contractuels venus d'Afrique, d'Inde et d'Asie. Cette période met en place les conditions sociales nécessaires à la pra-

\section{Les premiers clubs de football, créés à partir d'initiatives privées (1910), peinent à s'imposer face à la concurrence des gymnastiques patriotiques prônées par l’armée et l’Église.} tique des sports. La fin de l'esclavage et ses corollaires - la pacification de manière durable de la société, la promulgation de droits civiques, la représentation parlementaire - sont autant d'éléments sociopolitiques qui rendent possible une vie associative.

La seconde période correspond au développement des populations des îles de la Caraïbe. Un enracinement des populations dans les îles dû à l'accroissement naturel plus qu'à l'arrivée de nouveaux migrants. Cette période débute au premier tiers du vingtième siècle (1920-1930) et dure trente ans. Le vélo et l'escrime sont les premières pratiques sportives dans la colonie. Les premiers clubs de football, créés à partir d'initiatives privées (1910), peinent à s'imposer face à la concurrence des gymnastiques patriotiques prônées par l'armée et l'Église.

"Tous les gens chics de la colonie résident, quand ils le peuvent, à Saint-Claude où sont les plus belles villas. Beaucoup de ces demeures comportent un tennis. C'est même à peu près la seule trace d'accession au sport que j'ai vue dans nos Antilles, où le triomphant, l'universel football a à peine pénétré(1)." En 1931, cette vision immédiate d'un grand voyageur, le directeur de la Ligue maritime et coloniale française, Maurice Rondet-Saint, lors d'une visite dans la Caraïbe, atteste la marginalisation de la diffusion du football en Guadeloupe. 


\section{Les débuts du football dans la Caraïbe francophone}

Alors que le cricket se diffuse dans toutes les îles anglophones de la Caraïbe, le rugby et le football s'implantent au début du vingtième siècle en Haïti. Vers 1904, soit un siècle après l'indépendance, ce jeu, importé par des étudiants de retour après des études dans les universités européennes, débute à La Saline, dans l'ouest d'Haïti. Des jeunes fondent, en 1905, l'Union sportive haïtienne (USH). Les raisons pour lesquelles le football l'emporte sur le rugby sont peu connues. On peut supposer que l'action conjuguée des apports de jeunes plus influents, la construction du stade du Pont-Rouge et la création de nouveaux clubs ont été des éléments déterminants. Le premier match de football en Haiti oppose l'Union Sportive Haïtienne à l'Union Athlétique de Port-de-Paix. Les premières fédérations sportives s'organisent au sein de la Direction générale des sports en Haïti, de l'Union des sociétés sportives haïtiennes peu de temps après (1912), puis de la Fédération haïtienne de football amateur, l'actuelle Fédération haïtienne de football.

Le football forme les premiers grands champions internationaux haïtiens des années vingt notamment Silvio Cator (1900-1952). Il joue au Tivoli Athletic Club et au Racing Club haïtien, avant d'être sélectionné aux Jeux olympiques de Paris (1924) en saut en hauteur et en saut en longueur, et médaillé d'argent aux Jeux d'Amsterdam (1928). Il invente la technique moderne du double ciseau et bat le record du monde du saut en longueur le 9 septembre 1928 à Colombes, en réalisant 7,93 mètres. Sa carrière se termine aux Jeux olympiques de Los Angeles en 1932. À son retour en Haïti, il devient maire de Port-au-Prince en 1946, et "immortel" dans la mémoire collective de son île. L'actuel stade de Port-au-Prince, anciennement parc Leconte, porte son nom depuis 1952.

En 1934, la sélection nationale haïtienne participe à la Coupe du monde en Uruguay et devient ainsi la première équipe de la Caraïbe qualifiée à cet événement mondial. De la "perle des Antilles" viennent deux figures marquantes de la compétition mondiale. Joseph Édouard Gaetjens (1924-1964), né à Port-au-Prince d'une mère haïtienne et d'un père belge, porte le maillot de l'équipe des États-Unis lors de la Coupe du monde de 1950 au Brésil. Son but de la victoire contre l'Angleterre (1-0) reste à jamais dans l'histoire du football transatlantique. Emmanuel Sanon (1951-2008), dit "Manno", est le seul buteur haïtien en Coupe du monde ( 2 buts). Son premier but au stade de Munich en 1974 achève une série de 19 matchs officiels durant lesquels le portier italien Dino Zoff n'avait encaissé aucun but. Sanon passe six saisons au Germinal Beerschot d'Anvers, où il remporte la coupe de Belgique en 1979. 


\section{Le foyer guadeloupéen et la création des premières ligues de football}

Comparé à Haïti, le football en Guadeloupe se diffuse différemment sur d'autres logiques migratoires, plus complexes. Durant les années vingt, les premiers étudiants guadeloupéens de retour de métropole, les fonctionnaires français ainsi que les nouveaux migrants italiens, libanais, syriens, véhiculent de nouveaux apports culturels. Ces hommes en recherche d'intégration sociale contribuent à la création des premiers grands clubs de football à Basse-Terre et Pointe-à-Pitre.

Dans les milieux bourgeois blancs et mulâtres de Basse-Terre, le Racing Club ${ }^{(2)}$ est créé en 1924. La scission de ce club en raison de tensions raciales provoque la création de deux clubs en 1930 : le Cygne Noir et la Gauloise. À Pointe-à-Pitre, les logiques sociales et raciales se reproduisent dans les plus grands clubs. Les Sonis constituent le club des catholiques ; la Solidarité scolaire (1917) regroupe une bourgeoisie intellectuelle noire ; les mulâtres s'organisent au Redoutable ; les classes populaires quant à elles se retrouvent en forte proportion au Red Star et à l'Athlétique Club Darboussier, le club de la plus grande usine de l'île. 
En 1923, durant ses études, Édouard Chartol, un mulâtre, fonde à Paris, avec un Martiniquais, le docteur Zizine, et un autre Guadeloupéen, Gérard Alfred, le Racing Club Antillais ${ }^{(3)}$. Ce club rassemble des ressortissants coloniaux - des étudiants essentiellement - et exprime dans le football national une affirmation identitaire. Edouard Chartol retourne dans la colonie en 1928. En 1929, il crée un organisme omnisports, le Club guadeloupéen des sports généraux, puis la Fédération guadeloupéenne des sports athlétiques ${ }^{(4)}$. Deux structures voient le jour sous son impulsion, la Ligue sportive de la Grande-Terre(LSGT) en 1931 à Pointeà-Pitre et l'Union sportive basse-terrienne (USBT) en 1933 à Basse-Terre. Les deux organismes fédérateurs fusionnent en 1939 dans la Fédération sportive de la Guadeloupe (FSG), chargée d'organiser les épreuves pour l'ensemble du territoire. Ces ligues développent une rivalité brutale, à caractère raciste, entretenue par une opposition "de clochers" entre Pointe-à-Pitre et Basse-Terre. Il faut attendre 1936 et l'arrivée du gouverneur du Front populaire, le Guyanais Félix Éboué, pour initier la construction des premiers stades réglementaires, un à Basse-Terre, le stade Éboué, et l'autre à Pointe-à-Pitre, le stade Darboussier.

\section{La Carailbe, premier lieu d'expression internationale du football guadeloupéen}

Le football guadeloupéen s'organise en marge du football français : la métropole ne porte aucun intérêt aux sportifs coloniaux, d'autant qu'ils n'apportent aucun prestige à la nation. Le jeu se popularise progressivement par l'action de la presse, des mécènes et par l'activité des prosélytes. La Caraïbe est le lieu d'expression des premiers footballeurs guadeloupéens. En 1946, des sportifs et des dirigeants de la Dominique (Dominica Amateur Sport Association) ${ }^{(5)}$ visitent la Guadeloupe ; en 1947, le Violette Athlétique Club d'Haïti fait une tournée ${ }^{(6)}$. L'année suivante, la Guadeloupe organise la première compétition de football intercaribéenne francophone : le Trophée Caraïbe. Cet affrontement oppose quatre îles - Haïti, la Dominique, la Martinique, la Guadeloupe - et les trois Guyane (hollandaise, anglaise et française). Le 19 décembre 1948, la Guadeloupe remporte le Trophée ; elle défait en finale la Martinique avec 3 buts à 0 . Le capital symbolique de cette victoire offre aux Guadeloupéens une légitimité sportive dans la Caraïbe. À ce titre, en 1951 à Porto Rico, la Guadeloupe, représentée par Max Caberty et Gaston Adélaïde, devient membre fondateur de la Caribbean Football Association avec la Jamaïque, la République dominicaine, Haïti, Porto Rico, Trinidad, Aruba, la Guyane anglaise. Les deux frères Banguillot, Jack et Charles, sont sélectionnés dans 
la première équipe intercaribéenne. La sélection, dont Charles est le capitaine, dispute un tournoi en Jamaïque ${ }^{(7)}$.

La loi du 19 mars 1946 transformant la colonie en département a pour conséquence majeure d'amplifier les pressions exercées par la France pour la mise en conformité administrative des ligues sportives, sans tenir compte des réalités locales. En ce qui concerne le football, les discussions avec la Fédération française de football débutent en 1951 et s'achèvent en 1952 avec la création de la Ligue guadeloupéenne de football. La Guadeloupe se détourne de la Caraibe. Les compétitions s'orientent exclusivement vers la France, sans réels moyens et ni considération.

Bruno Dulac, professeur d'éducation physique guadeloupéen, président de l'association La Colombe, organise, en prenant tout à sa charge, le premier déplacement d'une équipe guadeloupéenne en France ${ }^{(8)}$. Le 3 juin 1952, l'équipe guadeloupéenne embarque sur le Colombie, pour une traversée de dix jours jusqu'au Havre.

Quatre matchs sont organisés contre trois clubs français et un club suisse. La Guadeloupe gagne un match contre l'Olympique Lyonnais, et perd les autres Olympique Lyonnais-Guadeloupe : 2-3 ; Le Havre-Guadeloupe : 2-1 ; ServetteGuadeloupe : 4-1 ; Red Star-Guadeloupe : 2-0.

Après cette compétition, trois joueurs tentent l'aventure à Lyon : Joseph Aucourt et les frères Banguillot. Jean Sorel, lui, fait carrière dans le sud-est de la France et reçoit des propositions du Real Madrid. Cette initiative privée laisse place à une émigration massive organisée par l'État dans les années soixante.

\section{L'émigration caribéenne francophone de 1960-1980}

De 1920 à 1960, la population de la Guadeloupe double. Elle passe de 154755 à 281640 habitants $^{(9)}$, ce qui représente un fort potentiel migratoire, tant en nombre d'effectifs qu'en caractéristiques sociales : une population jeune et sousqualifiée. La pression démographique de l'après-guerre devient considérable. L'économie locale, incapable de se diversifier après le déclin des plantations, ne peut pas absorber dans le marché du travail les générations de plus en plus nombreuses issues de cette "explosion démographique". Cet argument prédispose la mise en place d'une politique migratoire. Les pouvoirs publics, confrontés aux premiers conflits sociaux liés à la fermeture des usines sucrières et au mécontentement général (la montée des nationalismes), assimilent le problème du développement de la Guadeloupe et de la Martinique à une question d'équilibre démographique. Cette émigration massive favorise des chômeurs appelés à 
occuper des emplois peu qualifiés dans la fonction publique (Assistance publique, PTT) à laquelle la main-d'ceuvre étrangère ne pouvait prétendre. À cela s'ajoutent des incitations diverses, l'Armée, les créations d'organismes spécialisés, le Bumidom $^{(10)}$. Le développement considérable des transports aériens et l'attrait mythique de niveaux de vie supérieurs pour les migrants facilitent les départs ${ }^{(11)}$. La vie en France était réputée plus facile, d'autant que l'on croyait que la citoyenneté française était plus déterminante que l'appartenance ethnique et ses corollaires culturels. L'acquisition de la culture française était identifiée à l'ascension sociale et celle-ci à la mobilité géographique, avec pour principe : " $L a$ migration est une chance pour les Antilles ${ }^{(12)}$ ?'

\section{Le foot à l'épreuve des communautarismes}

À partir des années soixante-dix, les possibilités d'emplois diminuent. La "migration organisée" entame un net recul : de 35621 migrants de 1962 à 1965 on passe à 1426 pour l'année 1981 , soit une baisse de près de $25 \%$. Cependant, les problèmes d'insertion sociale des migrants d'outre-mer et la permanence des crises structurelles des îles suscitent un renversement de la tendance. En France, les Guadeloupéens et les Martiniquais prennent conscience du fait que la couleur de peau transforme un Français de couleur en étranger. Les "domiens" se considèrent comme des "Français à part entière devenus des Français entièrement à part". Cette perception de "Français entièrement à part" renforce l'adhésion des ressortissants d'outre-mer aux sentiments communautaristes et pose concrètement le problème de l'intégration des populations ultramarines. Cette subjectivité s'objective dans la statistique nationale par un traitement spécifique des hommes : l'existence d'une catégorie particulière, les originaires des "DOM-TOM" dotée de caractéristiques sociologiques proches des immigrés ${ }^{(13)}$ : une population jeune, résidant dans les habitations à loyers modérés des zones défavorisées (les quartiers), fortement touchée par le chômage, dotée de faibles capitaux scolaires, sur laquelle s'exerce une forte discrimination raciale ${ }^{(14)}$. On comprend aussi le rôle de la sélection sociale inconsciente à laquelle sont soumis les meilleurs sportifs guadeloupéens. Tout est mis en place pour que la pratique du sport, notamment celle du football, paraisse de manière inconsciente la seule chance de réalisation sociale pour les jeunes les plus démunis. L'attrait d'une carrière sportive amène bon nombre d'entre eux, encouragés par leurs professeurs, leurs parents et leur entourage, à placer tous leurs espoirs dans le sport professionnel. 


\section{Quand les joueurs deviennent des symboles}

La politique migratoire révèle dans le domaine du sport une masse critique de pratiquants en métropole. Autrement dit, elle rend visible de manière progressive un plus grand nombre de sportifs talentueux des anciennes colonies dans les équipes de France. Ces internationaux contribuent activement à fabriquer de nouvelles conceptions dans la manière de penser les identités insulaires et les solidarités ethniques et culturelles. Ils construisent un imaginaire collectif, replacé dans les effets symboliques de l'ensemble des migrations des années cinquante ${ }^{(15)}$. Le sens commun qualifie cette réalité sportive fortement appréciée des entraîneurs de "sport antillais". Arsène Wenges résume en partie cette spécificité française : "Nos joueurs noirs ont des qualités spécifiques. Là, on profite à la fois de l'immigration et de l'apport des excolonies. Tous les pays ne peuvent disposer aujourd'hui d'athlètes de cette qualité(16)." 
Lémigration exprime l'intensité des luttes que mènent les hommes avec leur corps pour l'accès aux pratiques, mais surtout leurs possibilités d'ascension sociale. Ces luttes dans le football débutent en 1931 avec le Guyanais Raoul Diagne, le premier Noir en équipe de France, qui joue une Coupe du monde en 1938. Le Martiniquais Xercès Louis joue à Lyon en 1941 et en équipe de France en 1954. D'autres luttes se mènent dans l'armée, à l'exemple de celle de Flavien Kancel, un militaire guadeloupéen qui joue dans l'équipe du Vietnam en plein conflit colonial ${ }^{(17)}$. À la même époque, l'Armée donnera à Jean Mathurin la possibilité de devenir le premier conseiller

Thierry Henry, enfant d'un Guadeloupéen et d'une Martiniquaise, est en fait un banlieusard né aux Ulis, où il grandit et débute sa carrière. technique de football de la Guadeloupe. D'autres joueurs talentueux évoluent en France, tels Albert Couriol et Luc Sonor, pour ne citer que les plus célèbres.

Mais c'est la carrière du Guadeloupéen Marius Trésor ${ }^{(18)}$ qui symbolise le plus cette période migratoire. Ses exploits ont pour effet symbolique de transformer de manière durable les représentations françaises. Marius Trésor débute dans le football à la Juventus de Sainte-Anne et sa carrière professionnelle dure quinze ans. Il devient, dans les années quatre-vingt, un modèle de réussite et d'identification pour la communauté antillaise et les jeunes footballeurs. Défenseur central intraitable, le plus réputé au niveau international, détenteur du record de sélections en équipe nationale, capitaine de l'équipe de France, Trésor construit un mythe grâce à ses exploits en Coupe du monde (1982).

\section{Le temps du "transbord" ou la rupture migratoire}

Le ralentissement de l'émigration n'empêche pas la communauté antillaise de s'accroître en France. Une nouvelle dynamique sociodémographique est à l'ceuvre, à laquelle les naissances en France d'enfants de parents nés dans les îles portent une contribution déterminante entre 1982 et 1990. Le nombre des naissances est supérieur aux nouvelles installations durables de migrants arrivant de la Caraïbe francophone. Les potentialités de croissance de la population des îles ne dépendent plus des seules potentialités de l'émigration au départ des Antilles. Ces naissances hexagonales transforment l'émigration de travail des Antillais en France en une immigration de peuplement, localisée fortement dans la région parisienne sur un axe nord/nord-est. En mars 1999, 357000 natifs des DOM-TOM habitaient en 
métropole ${ }^{(19)}$, soit légèrement moins que la population de la Guadeloupe (447 000). La constitution de cette population des originaires des "DOM-TOM" révèle des caractéristiques sociologiques propres.

Dans le football, ce fait s'incarne dans la présence d'un grand nombre de joueurs nés en France, évoluant dans les clubs et au plus haut niveau dans les clubs européens, qui revendiquent une identité autre que celle de leur lieu de naissance. Mikaël Silvestre, William Gallas et Thierry Henry illustrent cette tendance. Thierry Henry, enfant d'un Guadeloupéen et d'une Martiniquaise, est en fait un banlieusard né aux Ulis, où il grandit et débute sa carrière. Attaquant au FC Barcelone, après une carrière à Arsenal, Henry est considéré comme le meilleur attaquant de sa génération; actuellement, il est le meilleur buteur français de l'histoire, toutes compétitions confondues. Il revendique fortement ses origines insulaires et son attachement à la Guadeloupe, où il retourne souvent, à la Désirade : "Quand j'y débarque, jenlève alors mes chaussures et mon tee-shirt et je redeviens un gars de là-bas. Je pars me balader pied nus, 
je rencontre des gens qui me connaissent depuis que je suis tout petit ${ }^{(20)}$." William Gallas porte sa revendication dans l'action qu'il mène auprès des jeunes dans le football en organisant tous les ans un trophée en Guadeloupe : le Trophée Gallas.

\section{De nouvelles formes d'expression identitaires : les Gwada Boys}

En 2010, les footballeurs guadeloupéens sont reconnus bien au-delà de la Guadeloupe et de la France. C'est dans la Caraïbe que d'autres enjeux se jouent. L'éloignement du football guadeloupéen de la Caraïbe est dû en partie à la faible considération donnée aux voisins et surtout aux rapports de force politiques qui, de manière générale, prônent une assimilation du sport guadeloupéen au sport national. Ce rapport institutionnel empêche et limite les échanges internationaux. L'absence de considération des sportifs locaux au niveau national, les difficultés récurrentes rencontrées par les clubs, les ligues, le cadre compétitif restreint et le sous-développement des infrastructures amènent à partir des années quatre-vingt un retour dans la Caraibe. Les luttes personnelles menées alors par le docteur Jacques Rugard, président de la Ligue guadeloupéenne de football, aboutissent à l'entrée de la Guadeloupe dans l'Union de football de la Caraïbe. En 2007, aux États-Unis, la sélection guadeloupéenne s'incline 1 à 0 contre le Mexique en demi-finale de la Gold Cup. Cette sélection, les Gwada Boys ${ }^{(21)}$, menée par Jocelyn Angloma, un ancien international de retour en Guadeloupe, est renforcée par des joueurs guadeloupéens évoluant localement, en France : Stéphane Auvray, Aurélien Capoue, David Sommeil, et en Europe : Loïc Loval et Franck Grandel (FC Utrecht). Cette sélection a révélé contre toute attente les potentialités de l'équipe de la Guadeloupe, l'une des meilleures de la Caraibe. Plus que du sport, cette performance a cristallisé les passions autour d'une nouvelle identité recentrée sur le territoire insulaire : les Gwada Boys, nom donné par la presse américaine, récupéré et adopté par toute l'île. Tout en posant de graves questions institutionnelles et politiques - la participation de la Guadeloupe à une coupe intercontinentale, la libération des joueurs internationaux guadeloupéens évoluant dans les grands clubs français pour honorer une sélection régionale -, elle a donné de la fierté à toute une population et laisse entrevoir de nouvelles perspectives de pouvoir se penser et s'affirmer dans le football.

À ce titre, en 2008, les Gwada Boys, en se déplaçant au Sénégal dans le cadre de "Football et mémoire", constituent la première équipe antillaise à se déplacer en Afrique. En 2010, Stéphane Auvray, en signant aux Kansas City Wizards, dans la Major League Soccer (ligue professionnelle américaine), ouvre d'autres voies migratoires, en rupture avec les anciennes. 


\section{Notes}

1. Maurice Rondet-Saint, Des Antilles à Panama et à Costa-Rica, notes et croquis, Société d'éditions géographiques maritimes et coloniales, Paris, 1931.

2. M. Micaux, G. Huguette, D. Édouard, Le Racing Club de 1925 à 1987, document dactylographié, 1987.

3. Voir Le Bec du 24 avril 1936, "Un bienfaiteur au Bec" ; Le Bec du 5 juin 1936, "Le Docteur Zizine et le Racing-

Club Antillais" ; Le Bec du 14 mai 1937, "Le Racing Club Antillais à Bordeaux".

4. E. Chartol (1901-1992), ancien éclaireur de France et moniteur de la préparation militaire de 1916-1918.

5. Voir Le Dimanche sportif et culturel $n^{\circ} 32$ du 10 novembre 1946, "Deuxième visite en Guadeloupe des sportifs et touristes de la Dominique".

6. Voir Le Dimanche sportif et culturel du 4 mai 1947, "Reportage de la croisière sportive haïtienne", pp. 202-207.

7. Voir Le Dimanche sportif et culturel du 9 mars 1952, "Les frères Banguillot de retour de Jamaïque".

Composition de la sélection caribéenne:

Gardiens : Joey Gonsalves (Trinité), Mateo Reyes (Aruba.

Arrières : Gerry Parsons (Trinité), André Dieudonné (Haïti,) Manny Desouza (Guyane Anglaise).

Demis : Delbert Charleau (Trinité) Allan Joseph (Trinidad), Yvon Dorceans (Haïti), Rafael Baez Guillen (Porto-Rico), Humphey Mynals ( Surinam).

Avants : André Vieux (Haïti), Adrian Brokke (Aruba), Juan Briezen (Aruba), M Moore (Guyane anglaises), Paul Derosiers (Haïti), Michel Kruin (Surinam), Rudie Kamperveen (Surinam) Jack Banguillot, Charles Banguillot.

8. Voir à ce sujet H. P. Mephon, "La venue de la première équipe guadeloupéenne en France", Les enjeux des activités physiques et sportives dans la construction d'une culture identitaire guadeloupéenne, Thèse de troisième cycle de sociologie, université de Nantes, 2003, pp. 243-245.

La sélection de l'équipe guadeloupéenne de La Colombe :

Jean Seytor (Solidarité Scolaire), Anatole Doressamy (Club Sportif capesterrien), Pierre Fifi (Red Star), Roger Plantier et Etienne Palmin (Cygne noir), Jojo Bride, Gérard Casalis et Pierre André (Gauloise), Joseph Aucourt, Charles et Jack Banguillot (Racing), Ido Obidol, André Sannier, Jean Sorel et Jacques Pontremy (Club sportif moulien).

9. Source J.-P. Guengant, Insee.

10. La mise en ceuvre concrète de l'immigration des ressortissants des départements d'outre-mer a conduit la création d'un organisme ad hoc, le Bureau des migrations des départements d'outre-mer (Bumidom), par l'arrêté du 26 avril 1963. Société d'État dotée de la personnalité civile et de l'autonomie financière, le Bumidom est placé sous tutelle du ministère des Finances. Il se voit confier le soin de contribuer à résoudre les problèmes démographiques relatifs aux départements d'outre-mer. Ses missions consistent à informer les futurs migrants (sélection, mise en route et accueil des candidats), à trouver des formations professionnelles et à placer la main d'ceuvre migrante, à faciliter les implantations à caractère familial, à gérer des centres d'accueil et de transit, à coordonner les différents organismes concernés.

11. A. Anselin, L' Émigration antillaise en France, la troisième île, Paris, Karthala, 2000 ; Du bantoustan au ghetto, Paris, Editions Anthropos, 1979.

12. Paul Dijoud, secrétaire d'État aux Départements et Territoires d'outre-mer en 1981.

13. Voir à ce sujet C. Valentin-Marie, "Les populations des DOM-TOM, nées et originaires, résidant en France métropolitaine”, Résultats, Démographie et société n²4, Insee, 1993. Le moyen imaginé par C. Valentin-Marie consiste à reconstituer une origine par filiation sur une génération. Cependant, le procédé bute sur les limites imposées par le formatage initial des données : l'auteur indique que la définition donnée ici est avant tout subordonnée à l'information statistique disponible.

14. Source: "Les discriminations dans le monde du travail", Cahier de recherche n ${ }^{\circ} 171$, Crédoc, mai 2002.

15. Voir sur ces questions M. Barreaud, Élite sportive et immigration : les footballeurs professionnels étrangers en France et leur intégration dans la société, 1945-1992, thèse de doctorat, université de Reims, 1996 et E. Cashmore, Black Sportmen, London, Routledge \& Kegan Paul Ltd, 1982.

16. A. Wenger, "On a dix ans d'avance", L'Équipe du 19 juin 2000.

17. Voir à ce sujet H. P. Mephon, "Le seul joueur français sélectionné dans l'équipe du Vietnam", Corps et Société en Guadeloupe. Sociologie des pratiques de compétitions, Presses universitaires de Rennes, 2007.

18. Voir à ce sujet H. P. Mephon, "Marius Trésor", Corps et Société en Guadeloupe. Sociologie des pratiques de compétitions, Presses Universitaires de Rennes, 2007, p. 338.

19. Voir à ce sujet C. V. Marie, L. Qualité, "Un quart des personnes nées aux Antilles vit dans l'Hexagone", Antiane éco $\mathrm{n}^{\circ}$ 52, INSEE, mai 2002, pp. 15-18 ; C. V. Marie, S. Signori, "La population des DOM-TOM vivant en métropole", Insee-Première $\mathrm{n}^{\circ}$ 204, juin 1992.

20. "Henry, la France au cceur des Antilles", France football n ${ }^{\circ} 3109$ du 8 novembre 2005.

21. http://www.gwadaboys.net/gwadaboys.html 